one case occurred in the present series, and a patient of mine, a girl aged 16, developed the syndrome following irradiation of the neck for Hodgkin's disease.

The present authors point out that the early concepts of moyamoya disease as a relatively benign disorder in Japanese girls have been modified in recent reports. The sexes were equally affected and the majority of patients were Caucasian in their series. The clinical course depends on the rapidity and extent of vascular occlusion and the ability to develop a collateral circulation. The progressive nature and serious sequelae of cases left untreated are stressed.

\title{
ARTERIOVENOUS MALFORMATIONS OF THE BRAIN
}

Twenty-three children with A-V malformations have been treated by neurosurgeons at the Children's Hospital of Eastern Ontario, Ottawa, Ontario. Fourteen were boys and $\bar{g}$ giris. The averaje age at presentation was 10 years. The majority (83\%) presented with spontaneous hemorrhage and only one (4.3\%) with seizures. Angiography was performed in 21 patients. The AVM could not be demonstrated in 5 (24\%) who had an occult or cryptic AVM. Contrast-enhanced CT also failed to show abnormal vessels in 3 of the 5 occult AVMs.

Of 18 survivors, 15 were normal and 3 slightly disabled (2 with epilepsy). Aggressive surgical intervention resulted in improved survival and low morbidity. Overall mortality in the group was $22 \%$ while complete surgical excision carried a $7 \%$ mortality. The authors conclude that a spontaneous cerebral hemorrhage in a child is probably due to a vascular malformation, even when angiography and enhanced CT are negative. CT contrast enhancement is not a reliable indicator of occult AVM and direct surgery is needed for diagnostic confirmation and prevention of further hemorrhages. Also, children presenting with symptoms other than hemorrhage (e.g. seizures), should undergo surgery to prevent bleeding, providing the lesion is accessible with low risk to healthy brain. (Ventureyra ECG, Herder S. Child's Nerv Syst $1987 ; \underline{3}: 12-18$ ).

COMMENT: AVMs that bleed usually require neurosurgical management, and total excision seems the treatment preferred when feasible. Stereotaxic radiosurgery and the proton beam are available in some centers for cases not amenable to excision. The treatment of the AVM presenting with seizures or recurrent headaches but without spontaneous hemorrhage is often a neurologist's responsibility. When should he involve his neurosurgical colleague? In the past, neurologists have sometimes chosen conservative management. The present study argues in favor of surgery for all AVMs in children diagnosed radiologically, given accessibility and low risk to surrounding brain tissue. Even the neonate with the AVM that involves the vein of Galen and presents with congestive heart failure has a better chance of survival with surgery. (see HOFFMAN et al J Neurosurg $1982 ; 57: 316)$.

\section{CNS NEOPLASMS}

\section{PINEAL REGION TUMOURS OF CHILDHOOD}

At the University Hospital, Hamburg, from 1980-85, 17 children had pineal region tumours among 102 children with CNS tumours. The authors conclude: (1) that the incidence in Germany is higher than assumed and at least equal to that in Japan, (2) radiation without 